\title{
Benign Paroxysmal Vertigo of Childhood
}

\author{
Vishal Gurunath Pawar ${ }^{1}$ \\ ${ }^{1}$ Department of Neurology, Aster Specialist Medical Center, \\ International City, Dubai, United Arab Emirates
}

\begin{abstract}
Address for correspondence Vishal Gurunath Pawar, MBBS, DNB Medicine, DNB Neurology, SCE Neurology, Aster specialist medical center, Neurology clinic, International city, Dubai, United Arab Emirates (e-mail: neurovishal@gmail.com).
\end{abstract}

\begin{abstract}
Keywords

- paroxysmal vertigo of childhood

- BPV of childhood

- migraine variant

The aim of the article is to describe a case of benign paroxysmal vertigo (BPV) in childhood. It emphasizes the fact that every dizziness is not vertigo and every vertigo is not benign paroxysmal positional vertigo. BPV in childhood often goes unrecognized. This case report aims at creating awareness about this benign and self-limiting disorder.
\end{abstract}

\section{Introduction}

Benign paroxysmal vertigo (BPV) is a disorder of early childhood that was first described by Basser in $1964 .^{1}$

The BVP is characterized by recurrent episodes of brief disequilibrium. ${ }^{2}$ Typically, the onset is at 3 to 4 years of age. Sometimes, it can present late at the age of 7 or 8 years. ${ }^{3}$

The pathogenesis of BPV is not yet fully understood. ${ }^{3} \mathrm{BPV}$ typically occurs in healthy children without any history of major illness in the past. The attacks usually occur in clusters. The attacks in a cluster occur daily for several days, then it might remit for several weeks, and recur again as another cluster. ${ }^{2}$ There is no residual disability after the attacks. ${ }^{4-6}$

The semiology of the attack is, the child appears frightened and feels a spinning sensation, i.e., vertigo. He or she may reach out to maintain the balance. The attack may be associated with nystagmus and autonomic symptoms, such as nausea, vomiting, and diaphoresis. To prevent the fall, the older children might grab nearby persons or furniture for support. Episodes usually last less than a minute and are not associated with an altered consciousness. ${ }^{2}$

The otological, neurological, or radiological examination is normal. ${ }^{3}$ The caloric testing may show abnormal finding in the form of complete unilateral or bilateral canal paresis suggestive of vestibular dysfunction. This condition occurs in attacks and cease after the attacks. ${ }^{6,7}$ Imaging of the brain and EEG are normal. ${ }^{3}$

As per the current evidence, BPV is regarded as migraine equivalent. ${ }^{8-11} \mathrm{~A}$ large number of follow-up studies show that most of the children suffering from BPV develop some features typical of migraine during adulthood. ${ }^{4,8}$ Other studies show that most of the children have also a positive family history of migraine. ${ }^{6,12}$

\section{Case Report}

A 11-year-old boy came with history of attacks of vertigo from 4 years. He was apparently alright 4 years ago. The attacks are sudden, unprovoked, and not related to change in the body or head position. The boy gets frightened during the attack. Duration of each attack ranges in minutes. He gets clusters of the attacks ranging from 10 minutes to 5 hours in duration. The child becomes restless during the attacks and prefers not to sit at one place. He runs and grabs his parents. He cries and holds his parents tightly. Some of the attacks are associated with vomiting. The triggers for getting such attacks is playing for long hours, theme park rides, and sitting in a giant wheel. The child is well between the attacks. The attacks recur every 6 months.

He denies associated headache, tinnitus, deafness, photophobia, phonophobia during, after, or between the attacks. There is no history of loss of consciousness during or after the episodes. There is no history of otitis media. There is no personal or family history of epilepsy. The neurological and otological examinations are normal. The MRI brain and the EEG are normal. Patient was treated with (1) lifestyle modifications, (2) prophylactic medications, and (3) acute medications. 
(1) Lifestyle modifications: He was advised to keep a regular routine of his daily activities. He was also advised to keep same schedule of his meals and the sleep routine. He was advised to avoid lack of sleep, excess exposure to sunlight, loud sounds, strong smells, bright lights, etc.

(2) Prophylactic medications: He was given prophylaxis of migraine in the form of propranolol $5 \mathrm{mg}$ OD and magnesium supplementation.

(3) Acute medications: Patient reported that he feels better with betahistine for the acute attacks. It was started to him by his previous physician. He was advised to take the same.

\section{Discussion}

The benign paroxysmal vertigo is an important childhood disorder that needs early recognition. The counseling of the parents regarding the benign nature of the disorder helps to relieve their anxiety. The diagnosis of childhood BPV is established by the recurrent brief attacks of vertigo occurring without any warning, usually starting in the first decade of life in the absence of a significant medical history and resolving spontaneously.

Benign paroxysmal vertigo of childhood is regarded as a variant of migraine. ${ }^{13}$ It has been included in the recent International classification of headache disorders-3 (ICHD-3). ${ }^{13}$ The ICD code for the BPV is 1.6.2.

Following diagnostic criteria are proposed by the ICHD-3.

\section{Diagnostic Criteria}

A. At least five attacks fulfilling criteria B and C.

B. Vertigo* occurring without warning, maximal at onset and resolving spontaneously after minutes to hours without loss of consciousness.

C. At least one of the following associated symptoms or signs:
1. Nystagmus
2. Ataxia
3. Vomiting
4. Pallor
5. Fearfulness

D. Normal neurological examination and audiometric and vestibular functions between attacks

E. Not attributed to another disorder.**

The neurological, otological, and radiological examinations are normal. ${ }^{3}$ The caloric testing might show vestibular dysfunction. ${ }^{14}$ Horizontal semicircular canal paresis or

\footnotetext{
*Young children with vertigo may not be able to describe vertiginous symptoms. Parental observation of episodic periods of unsteadiness may be interpreted as vertigo in young children.

${ }^{* *}$ Posterior fossa tumors, seizures, and vestibular disorders must be excluded. The classification also mentions that the relationship between BVP and vestibular migraine needs to be further examined.
}

paralysis is seen in some children with BPV; in some, the excitability of the horizontal semicircular canal remains normal. ${ }^{15,16}$ We did not use the vestibular evoked myogenic potential (VEMP) and caloric testing during the diagnostic procedure in this patient. The dysfunction of the VEMP indicates saccular dysfunction. Dysfunction of the VEMP in BPV is described by some authors. ${ }^{7,17,18}$ There is no explanation of this phenomenon in the literature. ${ }^{3}$ Ischemia of vestibular pathways could be a trigger for the BPV attack as per some theories. ${ }^{17,19,20}$ One of the theories for the pathogenesis of migraine is cerebral vasospasms. If the vasospasm occurs in labyrinthine artery perfusion, it can cause ischemia of the inner ear and trigger the symptoms seen in the BPV. ${ }^{21-23}$ Transcranial doppler (TCD) measurements and high stimulus rate brainstem evoked potentials (BEPs) have further supported this theory. High stimulus rate BEPs indicates synaptic dysfunction. ${ }^{21}$ The TCD detects changes in cerebral blood flow velocity. ${ }^{24}$ Zhang et al in their study showed that the high stimulus rate BEP as well as the TCD was abnormal in half of the children suffering from BPV. ${ }^{18}$

\section{Conclusion}

Benign paroxysmal vertigo is an important disorder of the childhood that needs clinical suspicion. Early recognition, explanation of the benign nature, and spontaneous resolution of the disorder help to reduce the parental anxiety. It is widely accepted that the BPV is a migraine variant. Pathogenetic mechanism of vasospasm seen in migraine plays an important role in the pathogenesis of BPV of childhood.

\section{Conflict of Interest}

None declared.

\section{References}

1 Basser LS. Benign Paroxysmal Vertigo of Childhood. (A Variety of Vestibular Neuronitis). Brain 1964;87:141-152

2 Theresa Walls. Evaluation of dizziness in children and adolescents. In: UpToDate, Post, TW (Ed), UpToDate, Waltham, MA 2018

3 Kostić M, Trotić R, Jankes KR, Leventić M. Benign paroxysmal vertigo of childhood. Coll Antropol 36;2012(3):1033-1036

4 Schuknecht HF, Witt RL. Acute bilateral sequential vestibular neuritis. Am J Otolaryngol 1985;6(4):255-257

5 Lanzi G, Balottin U, Fazzi E, Tagliasacchi M, Manfrin M, Mira E. Benign paroxysmal vertigo of childhood: a long-term follow-up. Cephalalgia 1994;14(6):458-460

6 Russell G, Abu-Arafeh I. Paroxysmal vertigo in childrenan epidemiological study. Int J Pediatr Otorhinolaryngo 1999;49(Suppl 1):S105-S107

7 Chang $\mathrm{CH}$, Young YH. Caloric and vestibular evoked myogenic potential tests in evaluating children with benign paroxysma vertigo. Int J Pediatr Otorhinolaryngol 2007;71(3):495-499

8 Mira E, Piacentino G, Lanzi G, Balottin U, Fazzi E. Benign paroxysmal vertigo in childhood: a migraine equivalent ORL J Otorhinolaryngol Relat Spec 1984;46(2):97-104 $10.1159 / 000275693$

9 Abu-Arafeh I, Russell G. Paroxysmal vertigo as a migraine equivalent in children: a population-based study. Cephalalgia 1995;15(1):22-25, discussion 4 
10 Lewis DW. Migraine and migraine variants in children and adolescents. Semin Pediatr Neurol 1995;2(2):127-143

11 Mora R, Dellepiane M, Barettini L, Crippa B, Salami A. Electronystagmography in migraine equivalent syndrome. Otolaryngol Head Neck Surg 2009;140(4):566-572

12 Zhang D, Fan Z, Han Y, et al. Benign paroxysmal vertigo of childhood: diagnostic value of vestibular test and high stimulus rate auditory brainstem response test. Int J Pediatr Otorhinolaryngol 2012;76(1):107-110 10.1016/j.ijpsorl.2011.10.013

13 The International Classification of Headache Disorders. 3rd ed. Cephalalgia 2018;38(1)1-211

14 Finkelhor BK, Harker LA. Benign paroxysmal vertigo of childhood. Laryngoscope 1987;97(10):1161-1163

15 Marcelli V, Piazza F, Pisani F, Marciano E. Brain Dev 2006;28:80 DOI: 0.1016/j. braindev.2005.05.003

16 Gruber M, Cohen-Kerem R, Kaminer M, Shupak A. Vertigo in children and adolescents: characteristics and outcome. Scientific World Journal 2012;2012:109624

17 Lin KY, Hsu YS, Young YH. Brainstem lesion in benign paroxysmal children: evaluated by a combined ocular and cervical vestibular-evoked myogenic potential test. Int J Pediatr Otorhinolaryngol 2010;74(5):523-527.
18 Zhang D, Fan Z, Han Y, et al. Benign paroxysmal vertigo of children: diagnostic value of vestibular test and high stimulus rate auditory brainstem response test. Int J Pediatr Otorhinolaryngol 2012;76(1):107

19 Weiller C, May A, Limmroth V, et al. Brain stem activation in spontaneous human migraine attacks. Nat Med 1995;1(7):658-660

20 Jahn K. Vertigo and balance in children-diagnostic approach and insights from imaging. Eur J Paediatr Neurol 2011;15(4):289-294

21 Fuse T. ABR findings in vertebrobasilar ischemia. Acta Otolaryngol 1991;111(3):485-490

22 Huppert D, Strupp M, Theil D, Glaser M, Brandt T. Low recurrence rate of vestibular neuritis: a long-term follow-up. Neurology 2006;67(10):1870-1871

23 O'Reilly RC, Greywoode J, Morlet T, et al. Comprehensive vestibular and balance testing in the dizzy pediatric population. Otolaryngol Head Neck Surg 2011;144(2):142-148

24 Sloan MA, Burch CM, Wozniak MA, et al. Transcranial Doppler detection of vertebrobasilar vasospasm following subarachnoid hemorrhage. Stroke 1994;25(11):2187-2197 